\title{
Combination of SNX-2112 with 5-FU exhibits antagonistic effect in esophageal cancer cells
}

\author{
YUTING LIU $^{1 *}$, XIAO WANG ${ }^{1 *}$, YING WANG $^{2}$, YI ZHANG $^{1}$, KAI ZHENG $^{3}$, HAIZHAO YAN $^{1}$, LI ZHANG $^{1}$, \\ WENBO CHEN $^{1}$, XIAOYAN WANG ${ }^{1}$, QIUYING LIU ${ }^{1}$, SHAOXIANG WANG ${ }^{3}$ and YIFEI WANG ${ }^{1}$ \\ ${ }^{1}$ Guangzhou Jinan Biomedicine Research and Development Center, \\ National Engineering Research Center of Genetic Medicine, \\ ${ }^{2}$ Department of Biotechnology, Jinan University, Guangzhou 510632; \\ ${ }^{3}$ School of Medicine, Shenzhen University, Shenzhen 518060, P.R. China
}

Received August 13, 2014; Accepted September 24, 2014

DOI: $10.3892 /$ ijo.2014.2714

\begin{abstract}
The low efficacy of single-drug chemotherapy forms the basis for combination therapy in esophageal squamous cell carcinoma. SNX-2112, a selective heat shock protein 90 (Hsp90) inhibitor, was recently reported as being effective in combination with cisplatin and paclitaxel. In this study, we investigated the effect of SNX-2112 in combination with 5-fluorouracil (5-FU), another first-line anticancer drug, in esophageal cancer. Unexpectedly, tetrazolium assay revealed that the combination of SNX-2112 with 5-FU exhibited antagonistic effect. Flow cytometry revealed that the SNX-2112 and 5-FU combination greatly decreased the number of $\mathrm{G} 2 / \mathrm{M}$ cells compared to SNX-2112-only treatment in Eca-109 cells. This effect might be related to the altered mRNA level of cyclin-related genes including cyclin D1, Chk2 and Cdk4. Further, 5-FU attenuated SNX-2112-induced apoptosis by decreasing poly(ADP-ribose) polymerase (PARP) cleavage and inactivating caspase-3, -8 and -9. Additionally, 5-FU suppressed the SNX-2112-induced decrease of mitochondrial membrane potential. Moreover, 5-FU partly recovered Hsp90 client proteins, including Akt, p-Akt, inhibitor of $\kappa \mathrm{B}$ kinase (IKK) $\alpha$, extracellular signal-regulated kinase (ERK)1/2, and glycogen synthase kinase (GSK)-3 $\beta$, which SNX-2112 had downregulated. Taken together, this is the first report that the combination of SNX-2112 with 5-FU exhibited antagonistic effect in esophageal cancer cells by affecting growth inhibition, cell cycle, apoptosis, and Hsp90
\end{abstract}

Correspondence to: Professor Yifei Wang, Guangzhou Jinan Biomedicine Research and Development Center, National Engineering Research Center of Genetic Medicine, Jinan University, Guangzhou 510632, P.R. China

E-mail: twang-yf@163.com

Dr Shaoxiang Wang, School of Medicine, Shenzhen University, Shenzhen 518060, P.R. China

E-mail: hackerwsx@163.com

${ }^{*}$ Contributed equally

Key words: heat shock protein 90, SNX-2112, 5-fluorouracil, antagonism, apoptosis client proteins, suggesting that care is required in the clinical application of combined SNX-2112 and 5-FU.

\section{Introduction}

Despite significant reductions in esophageal cancer rates in association with lifestyle changes, esophageal cancer mortality remains high worldwide, and incidence, especially that of esophageal squamous cell carcinoma (ESCC) is increasing in China $(1,2)$. Chemotherapy is the most common treatment option for improving the poor survival rate in advanced esophageal cancer (3). The first-line drugs in the clinical treatment of esophageal cancer are, sequentially, cisplatin, 5-fluorouracil (5-FU), and paclitaxel (3). However, single-agent chemotherapy is not effective for esophageal cancer because of natural resistance and the development of resistance (4). In esophageal cancer, many studies have demonstrated that combination therapy is more effective than single-drug therapy $(3,5,6)$.

Several heat shock protein 90 (Hsp90) inhibitors that bind to the N-terminal ATP pocket of Hsp90 have entered clinical trials. Hsp90 is a crucial molecular chaperone for protein folding and stabilization that is considered a promising target for anticancer therapy (7). Hsp90 may be a target for esophageal cancer treatment because it and its client proteins are always overexpressed in several ESCC cell lines and patient tissues (8). Previously, we reported that the novel Hsp90 inhibitor BJ-B11 had potent antitumor activity by inducing cell cycle arrest, apoptosis, and autophagy in human ESCC Eca-109 cells (9). Liu et al reported that Hsp90 antisense RNA led to cell cycle changes and increased sensitivity to various chemotherapeutic agents in the same cell line (10). Wu et al suggested that 17-AAG, a traditional Hsp90 inhibitor, effectively inhibited proliferation and viability in other ESCC lines (11). Similarly, NVP-AUY922, another novel Hsp90 inhibitor, was a potent inhibitor of proliferation in esophageal cancer TE-4 cells (12). These reports provide a rationale for current preclinical efforts targeting Hsp90 to treat ESCC.

SNX-2112, a selective Hsp90 inhibitor, has broad antitumor activity and has entered phase I clinical trials in solid tumors and lymphoma (13). In previous studies, we identified the anticancer effect of SNX-2112 in breast cancer, hepatocel- 
lular carcinoma, and leukemia (14-16). Recently, SNX-2112 was reported as being effective in combination with cisplatin or paclitaxel in head and neck SCC (17). However, there have been no reports on SNX-2112 in combination with 5-FU, another widely used anticancer drug, in esophageal cancer. We examined the effects of SNX-2112 combined with 5-FU in esophageal cancer Eca-109 cells by detecting cell growth, cell cycle, apoptosis, and Hsp90 client proteins. This study may provide guidance for the clinical application of Hsp90 inhibitors.

\section{Materials and methods}

Reagents. SNX-2112 was synthesized as previously described in our lab with $>98.0 \%$ purity; we stored $10 \mathrm{mM} \mathrm{SNX-2112}$ stock solution in dimethyl sulfoxide (DMSO) at $-20^{\circ} \mathrm{C}(18)$. We purchased 5-FU, 3-(4, 5-diethylthiazol-2-yl)-2, 5-diphenyl tetrazolium bromide (MTT) from Sigma-Aldrich (St. Louis, MO, USA). An Annexin V-fluorescein isothiocyanate/propidium iodide (FITC/PI) staining kit was purchased from Beyotime (Haimen, China). RPMI-1640 medium and DMEM were purchased from Gibco (Carlsbad, CA, USA). Heatinactivated fetal bovine serum (FBS) was provided by the Sijiqing Co. (Hangzhou, China). Antibodies against caspase-3, caspase-8, caspase-9, poly(ADP-ribose) polymerase (PARP), Akt, phosphorylated (p)-Akt, inhibitor of $\kappa B$ kinase (IKK), extracellular signal-regulated kinase $(E R K)_{1 / 2}$, glycogen synthase kinase (GSK)-3 $\beta$, and glyceraldehyde-3-phosphate dehydrogenase (GAPDH) were purchased from Cell Signaling Technology (Beverly, MA, USA).

Cell culture. We cultured Eca-109 and EC-9706 cells (Cell Bank of the Chinese Academy of Sciences, Shanghai, China) in RPMI-1640 or DMEM medium supplemented with $10 \%$ FBS and $100 \mathrm{U} / \mathrm{ml}$ penicillin/streptomycin in $5 \% \mathrm{CO}_{2}$ at $37^{\circ} \mathrm{C}$.

Cell viability assay. Cell viability was assessed with the MTT assay. Exponentially growing cells $(\sim 3,500 /$ well in $100 \mu 1$ medium) were plated in 96-well culture plates, cultured overnight, and incubated with a series of concentrations of SNX-2112 $(0-2 \mu \mathrm{M})$ or 5 -FU $(0-100 \mu \mathrm{g} / \mathrm{ml})$ for $48 \mathrm{~h}$. After adding $10 \mu 1$ MTT solution per well, the plates were incubated at $37^{\circ} \mathrm{C}$ for $4 \mathrm{~h}$, the medium removed, formazan crystals solubilized in $100 \mu \mathrm{l} \mathrm{DMSO} /$ well, and the absorbance values read at $570 \mathrm{~nm}$. The inhibition ratio was calculated as follows: $\left(\mathrm{A}_{\text {control }}-\mathrm{A}_{\text {treated }}\right) / \mathrm{A}_{\text {control }} \times 100 \%$, where $\mathrm{A}_{\text {treated }}$ and $\mathrm{A}_{\text {control }}$ are the absorbance of the treated and control groups after $48-\mathrm{h}$ incubation, respectively.

Calculation of the combination effect index. We determined the inhibitory effects of SNX-2112 and 5-FU using the MTT assay. We used the combination index (CI) described by Chou and Talalay for analysis (19), performed using CalcuSyn software (BioSoft, Oxford, UK). $\mathrm{Cl}<1$ indicates synergism; $\mathrm{CI}=1$ indicates summation; $\mathrm{CI}>1$ indicates antagonism (20).

Cell cycle analysis. Cells were exposed to $0.125 \mu \mathrm{M}$ SNX-2112 or $25 \mu \mathrm{g} / \mathrm{ml} 5-\mathrm{FU}$ alone or in combination for $48 \mathrm{~h}$, harvested in cold phosphate-buffered saline, fixed in $70 \%$ ethanol, stored overnight at $4^{\circ} \mathrm{C}$, and resuspended in $50 \mu \mathrm{g} / \mathrm{ml}$ PI staining reagent containing $100 \mu \mathrm{g} / \mathrm{ml}$ RNase and $0.1 \%$ Triton X-100 for $30 \mathrm{~min}$ in the dark. Cells were analyzed by flow cytometry (FACSCalibur; Becton-Dickinson, San Jose, CA, USA).

Quantitative real-time RT-PCR ( $Q-P C R)$. Total cellular RNA was extracted using TRIzol reagent (Invitrogen, Carlsbad, CA, USA) according to the manufacturer's instructions. Quantitative RT-PCR was carried out using a Chromo4 instrument (Bio-Rad, Richmond, CA, USA) and SYBR ${ }^{\circledR}$ Premix Ex $\mathrm{Taq}^{\mathrm{TM}}$ kit (Takara Bio, Otsu, Japan) to detect the mRNA level of cyclin D1, Cdk2, Cdk4, p53, Chk1 and Chk2, with GAPDH as a normalizing control. The specific PCR primer sequences of these genes were designed by Primer premier 5.0 software (Table I). Cycling conditions were: $95^{\circ} \mathrm{C}$ for $20 \mathrm{sec}$ to activate DNA polymerase, followed by 40 cycles of $95^{\circ} \mathrm{C}$ for $10 \mathrm{sec}$, $55^{\circ} \mathrm{C}$ for $20 \mathrm{sec}$, and $65^{\circ} \mathrm{C}$ for $30 \mathrm{sec}$. The relative changes in gene expression were calculated with the $2^{-\Delta \Delta \mathrm{Ct}}$ method, where $\Delta \Delta \mathrm{Ct}=\Delta \mathrm{Ct}$ (drugs treated) $-\Delta \mathrm{Ct}$ (control) for RNA samples.

Annexin V-FITC/PI analysis. Cells were exposed to the indicated concentrations of SNX-2112 or 5-FU alone or in combination for $48 \mathrm{~h}$, resuspended in $500 \mu \mathrm{l}$ incubation buffer containing Annexin V-FITC and PI, incubated in the dark for $15 \mathrm{~min}$, and analyzed by flow cytometry. Data acquisition and analysis were performed using a FACSCalibur flow cytometer with CellQuest software (Becton-Dickinson).

Mitochondrial membrane potential assay. Cells were cultured on glass cover slips and treated with SNX-2112 and 5-FU for $48 \mathrm{~h}$, then incubated in complete medium containing $10 \mu \mathrm{g}$ fluorescent lipophilic cationic JC-1 dye for $20 \mathrm{~min}$ at $37^{\circ} \mathrm{C}$ in the dark. The stained cells were washed twice with JC-1 buffer solution and examined by laser scanning confocal microscopy. We also analyzed the cells by flow cytometry. Data acquisition and analysis were performed in a FACSCalibur flow cytometer using CellQuest software. The loss of mitochondrial membrane potential (MMP) was quantified as the percentage of cells expressing JC-1 monomer fluorescence.

Western blotting. Cell were treated with SNX-2112 and 5-FU for $48 \mathrm{~h}$, harvested, and lysed in sodium dodecyl sulfate (SDS) lysis buffer (SDS:phenylmethylsulfonyl fluoride $=50: 1$ ) at $100^{\circ} \mathrm{C}$ for $20 \mathrm{~min}$. Lysates were clarified by centrifugation $(12,000 \mathrm{rpm})$ at $4^{\circ} \mathrm{C}$ for $15 \mathrm{~min}$ and the supernatant was collected. Equal amount of lysate (20-30 $\mu \mathrm{g})$ was denatured in 5X SDS sample buffer, resolved with 6-15\% SDS-polyacrylamide gel electrophoresis, transferred to polyvinylidene difluoride membranes, blocked with 5\% skimmed milk in Tris-buffered saline containing $0.1 \%$ Tween-20 (TBST) at room temperature for $1 \mathrm{~h}$, and probed with primary antibody (1:1,000-1:5,000) overnight at $4^{\circ} \mathrm{C}$. The membranes incubated with secondary antibody $(1: 5,000)$ for $1 \mathrm{~h}$ at room temperature. Protein bands were visualized using an enhanced chemiluminescence kit (Beyotime, Shanghai, China) and imaged by autoradiography. GAPDH was used as the loading control.

Docking assay. The affinity of 5-FU and SNX-2112 for Hsp90 was determined by molecular operating environment docking. We obtained the crystal structure of Hsp90 from the Protein Data Bank (PDB code: 3R92). The two drugs were docked to 
Table I. Q-PCR primers.

\begin{tabular}{lll}
\hline Name & \multicolumn{1}{c}{ Forward primer (5'- to 3') } & Reverse primer (5'- to 3') \\
\hline Cyclin D1 & GCCCTCGGTGTCCTACTTC & CTCCTCCTCGCACTTCTGTT \\
Cdk2 & TGCCTGATTACAAGCCAAGTT \\
Cdk4 & CAGCTACCAGATGGCACTTACA & GAGTCGAAGATGGGGTACTGG \\
Chk1 & AAACATACCTCAACCCTTGGA & CAAAGATACAGCCAACACTCCA \\
Chk2 & TTGGAAGTGGTGCCTGTG & CCTTTTGCCCCTTTCTTG \\
p53 & CTCCTCAGCATCTTATCCGAGT & GGTCTGCCTCTCTTGCTGAA \\
GAPDH & AACGGATTTGGTCGTATTGGG & GCTGTTCCGTCCCAGTAGATTA \\
\hline
\end{tabular}

Table II. Combination effects of SNX-2112 and 5-FU on Eca-109 cells

\begin{tabular}{|c|c|c|c|}
\hline SNX-2112 $(\mu \mathrm{M})$ & $5-\mathrm{FU}(\mu \mathrm{g} / \mathrm{ml})$ & $\mathrm{Fa}$ & CI \\
\hline \multirow[t]{4}{*}{0.03125} & 50 & 0.71 & $2.662 \pm 0.329$ \\
\hline & 25 & 0.46 & $3.558 \pm 0.477$ \\
\hline & 12.5 & 0.23 & $3.319 \pm 0.509$ \\
\hline & 6.25 & 0.56 & $0.789 \pm 0.204$ \\
\hline \multirow[t]{4}{*}{0.0625} & 50 & 0.71 & $2.647 \pm 0.318$ \\
\hline & 25 & 0.68 & $1.601 \pm 0.296$ \\
\hline & 12.5 & 0.64 & $1.074 \pm 0.226$ \\
\hline & 6.25 & 0.26 & $3.136 \pm 0.154$ \\
\hline \multirow[t]{4}{*}{0.125} & 50 & 0.68 & $3.317 \pm 0.436$ \\
\hline & 25 & 0.52 & $3.772 \pm 0.396$ \\
\hline & 12.5 & 0.67 & $1.068 \pm 0.347$ \\
\hline & 6.25 & 0.42 & $2.151 \pm 0.447$ \\
\hline \multirow[t]{4}{*}{0.25} & 50 & 0.64 & $3.389 \pm 0.209$ \\
\hline & 25 & 0.61 & $2.884 \pm 0.175$ \\
\hline & 12.5 & 0.71 & $1.074 \pm 0.375$ \\
\hline & 6.25 & 0.68 & $0.859 \pm 0.167$ \\
\hline \multicolumn{4}{|c|}{$\begin{array}{l}\text { Cells were cultured with various concentrations with } \mathrm{SNX}-2112 \\
(0.03125-0.25 \mu \mathrm{M}) \text { and } 5 \text {-FU }(6.25-50 \mu \mathrm{g} / \mathrm{ml}) \text { for } 48 \mathrm{~h} \text {. The combi- } \\
\text { nation index }(\mathrm{CI}) \text { and fraction affected }(\mathrm{Fa}) \text { values were determined } \\
\text { using the pre-described method }(19) \text {. When } \mathrm{CI}<1 \text { indicates syner- } \\
\text { gism; } \mathrm{CI}=1 \text { indicates summation; } \mathrm{CI}>1 \text { indicates antagonism. Values } \\
\text { are mean } \pm \mathrm{SD} \text { (three independent experiments). }\end{array}$} \\
\hline
\end{tabular}

the binding pocket of Hsp90 to check the fitness according to our previous methods. A lower score indicated more favorable binding.

Statistical analysis. Data are expressed as the means \pm SD. Differences between two groups were analyzed using the Student's t-test; groups of three or more were analyzed using one-way analysis of variance (multiple comparisons). $\mathrm{P}<0.05$ and $\mathrm{P}<0.01$ were considered statistically significant. We performed statistical analyses using SPSS 17.0 software (IBM, Armonk, NY, USA).

\section{Results}

Effects of SNX-2112 and 5-FU on Eca-109 cells. Initially, we examined the effects of SNX-2112 and 5-FU on Eca-109
Table III. Combination effects of SNX-2112 and 5-FU on EC-9706 cells.

\begin{tabular}{lccc}
\hline SNX-2112 $(\mu \mathrm{M})$ & 5-FU $(\mu \mathrm{g} / \mathrm{ml})$ & $\mathrm{Fa}$ & $\mathrm{CI}$ \\
\hline 0.0625 & 25 & 0.71 & $3.366 \pm 0.429$ \\
& 12.5 & 0.67 & $2.102 \pm 0.377$ \\
& 6.25 & 0.68 & $1.079 \pm 0.234$ \\
& 3.125 & 0.42 & $1.75 \pm 0.404$ \\
0.125 & 25 & 0.89 & $1.276 \pm 0.213$ \\
& 12.5 & 0.71 & $1.853 \pm 0.407$ \\
& 6.25 & 0.63 & $1.563 \pm 0.326$ \\
0.25 & 3.125 & 0.53 & $1.453 \pm 0.454$ \\
& 25 & 0.68 & $4.355 \pm 0.236$ \\
& 12.5 & 0.83 & $1.143 \pm 0.234$ \\
& 6.25 & 0.76 & $1.107 \pm 0.145$ \\
0.5 & 3.125 & 0.70 & $1.102 \pm 0.214$ \\
& 25 & 0.86 & $1.869 \pm 0.345$ \\
& 12.5 & 0.90 & $0.865 \pm 0.209$ \\
& 6.25 & 0.82 & $1.118 \pm 0.375$ \\
& 3.125 & 0.75 & $1.38 \pm 0.334$ \\
& & &
\end{tabular}

Cells were cultured with various concentrations with SNX-2112 $(0.03125-0.25 \mu \mathrm{M})$ and 5-FU $(6.25-50 \mu \mathrm{g} / \mathrm{ml})$ for $48 \mathrm{~h}$. The combination index $(\mathrm{CI})$ and fraction affected $(\mathrm{Fa})$ values were determined using the pre-described method. When $\mathrm{CI}<1$ indicates synergism; $\mathrm{CI}=1$ indicates summation; $\mathrm{CI}>1$ indicates antagonism. Values are mean \pm SD (three independent experiments).

and EC-9706 cell growth by MTT assay. Cells were cultured in a range of concentrations of SNX-2112 or 5-FU for $48 \mathrm{~h}$. As expected, both SNX-2112 and 5-FU inhibited Eca-109 and EC-9706 cell growth in a dose-dependent manner. The median inhibitory concentration $\left(\mathrm{IC}_{50}\right)$ of SNX-2112 and 5-FU was $0.12 \pm 0.01 \mu \mathrm{M}$ and $48 \pm 0.2 \mu \mathrm{g} / \mathrm{ml}$ in Eca-109 cells, respectively (Fig. 1A). Similarly, the $\mathrm{IC}_{50}$ of SNX-2112 and 5-FU was $0.13 \pm 0.02 \mu \mathrm{M}$ and $3 \pm 0.2 \mu \mathrm{g} / \mathrm{ml}$ in EC-9706 cells, respectively (Fig. 1B). Unexpectedly, there was an antagonistic effect in most cases in the combination group (CI $>1$, Fig. 1C). The occurrences of strong antagonistic effect $(\mathrm{CI}>2)$ were $62.5 \%$ in Eca-109 cells and $18.75 \%$ in EC-9706 cells, respectively, suggesting that the antagonistic effect in Eca-109 cells was more obvious (Tables II and III). The effect was most obvious when $0.125 \mu \mathrm{M}$ SNX-2112 was combined with $25 \mu \mathrm{g} / \mathrm{ml}$ 5-FU in Eca-109 cells (CI=3.7, Table II and Fig. 1D). Therefore, we 

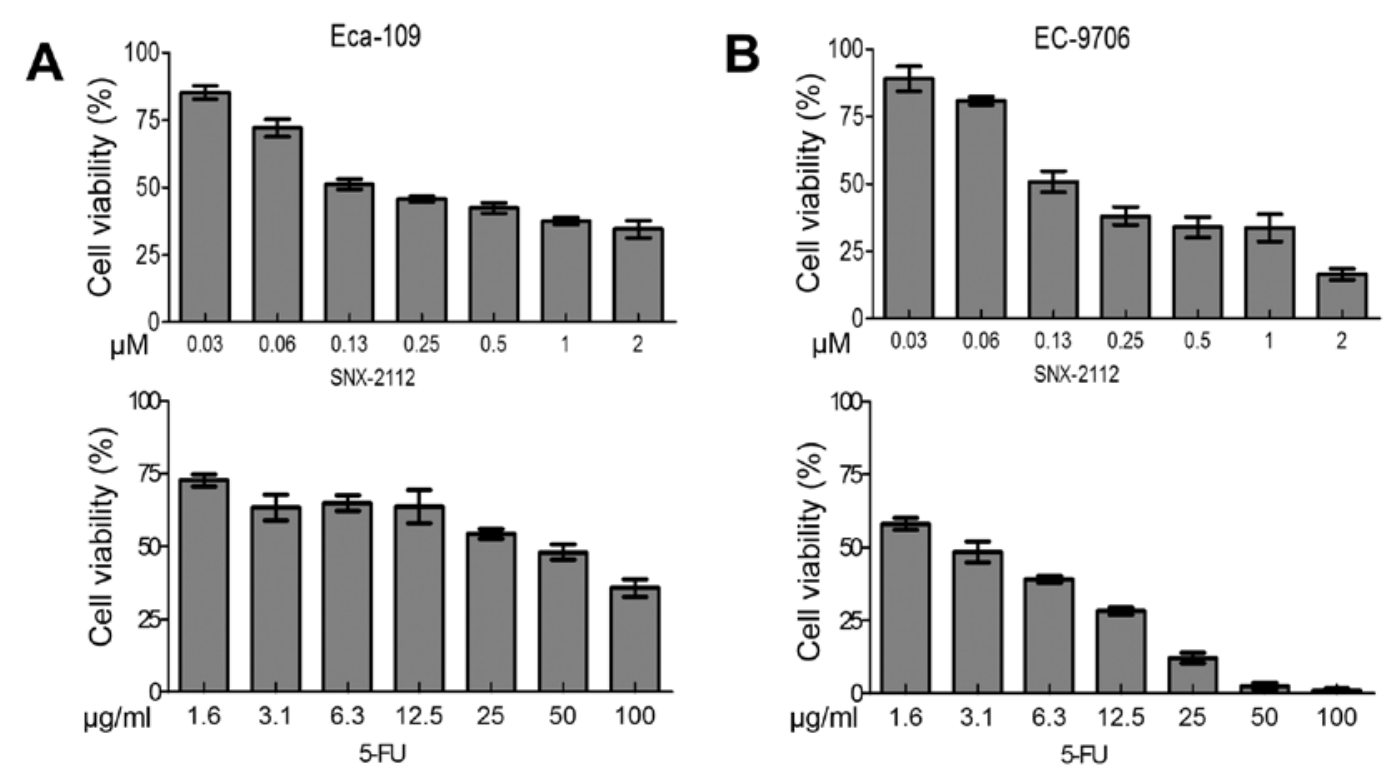

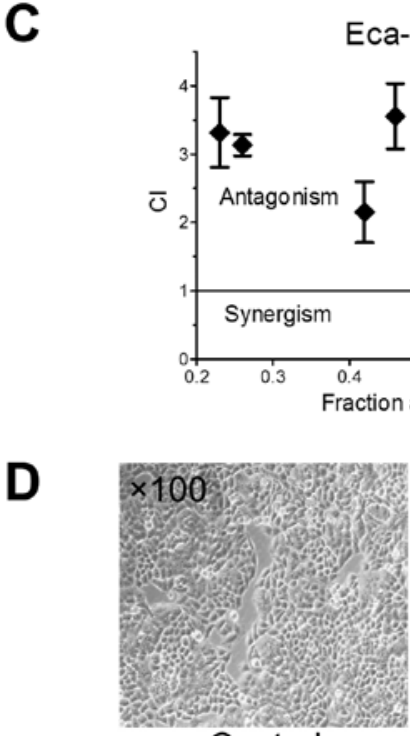

Control

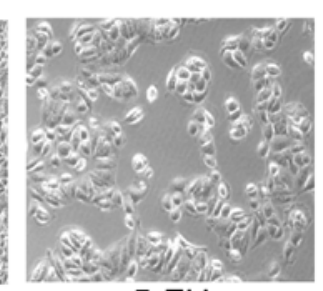

$5-F U$
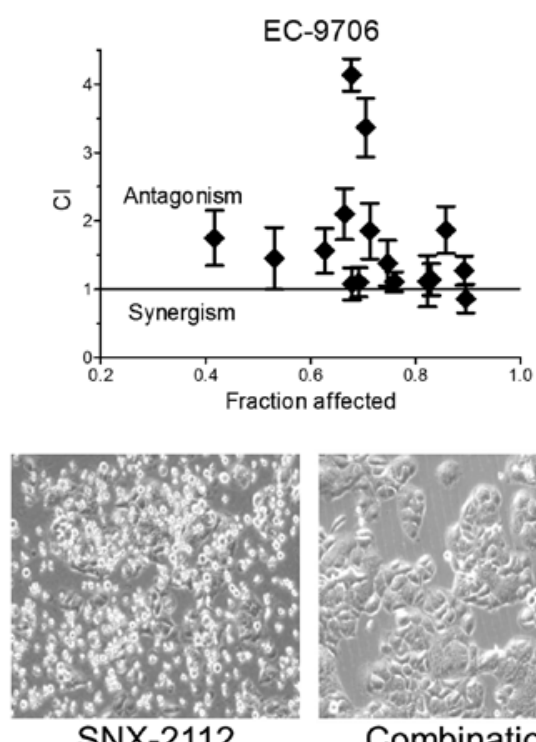

SNX-2112

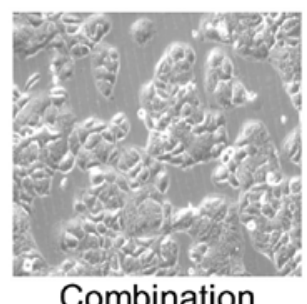

Combination

Figure 1. Effects of SNX-2112 and 5-FU on human esophageal cancer cell line. (A) MTT assay evaluating viability of Eca-109 cells treated with SNX-2112 (or 5-FU) alone for $48 \mathrm{~h}$. (B) MTT assay evaluating viability of EC-9706 cells treated with SNX-2112 (or 5-FU) alone for $48 \mathrm{~h}$. (C) The combination effects index of SNX-2112 and 5-FU in Eca-109 and EC-9706 cells. (D) Micrographs of Eca-109 cells after 5-FU, SNX-2112, or SNX-2112 and 5-FU treatment.

used $0.125 \mu \mathrm{M}$ SNX-2112 and $25 \mu \mathrm{g} / \mathrm{ml} 5-\mathrm{FU}$ as the optimal concentrations in Eca-109 cells for the remaining experiments.

5-FU blocked SNX-2112-induced G2/M arrest. Cell cycle arrest is the basic mechanism of cancer cell growth inhibition by Hsp90 inhibitors (16). To investigate the mechanism of 5-FU antagonism of SNX-2112 further, we examined cell cycle distribution with flow cytometry. There was $45.8 \%$ G2/M arrest and only $14.1 \%$ G0/G1 arrest following SNX-2112 treatment, but there was only $1.5 \% \mathrm{G} 2 / \mathrm{M}$ accumulation and the G0/G1 arrest increased to $48.2 \%$ in the combination group (Fig. 2A). This indicated that 5-FU completely recovered SNX-2112induced G2/M arrest and partly increased the G0/G1 arrest of SNX-2112. Further, we found that the mRNA levels of cyclin D1, Cdk4 and Chk2 were decreased in the combination group compared to treatment with SNX-2112 alone (Fig. 2B), while the mRNA levels of p53, Chk1 and Cdk2 did not change significantly (Fig. 2C).
5-FU suppressed SNX-2112-induced caspase-dependent apoptosis. To determine the effect of SNX-2112 plus 5-FU on apoptosis, we examined the ratio of apoptosis using flow cytometry. We found that SNX-2112 and 5-FU alone induced 47.02 and $20.71 \%$ apoptosis, respectively; SNX-2112 plus 5-FU led to apoptosis decreasing to $16.98 \%$ (Fig. 3A). We then examined caspase- 3 and PARP expression (indicators of apoptosis). 5-FU suppressed the caspase-3 downregulation and PARP cleavage induced by SNX-2112 (Fig. 3B). Taken together, our results indicate that 5-FU inhibits SNX-2112induced apoptosis and caspase activation in Eca-109 cells.

5-FU prevents the initial decrease in MMP during SNX-2112-induced apoptosis. Mitochondria play a central role in determining cell survival or response to diverse stimuli (21). The SNX-2112 and 5-FU combination greatly suppressed the caspase- 9 activity (a downstream indicator of the mitochondrial apoptotic pathway) induced by SNX-2112, 

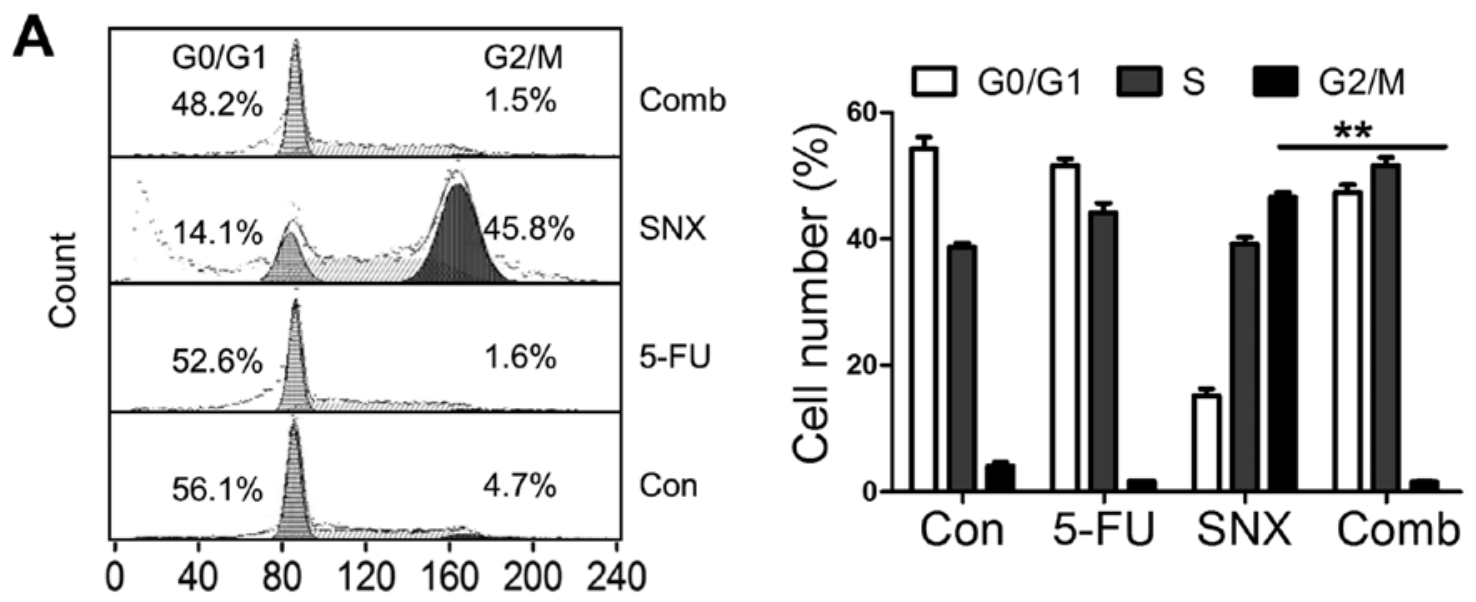

B
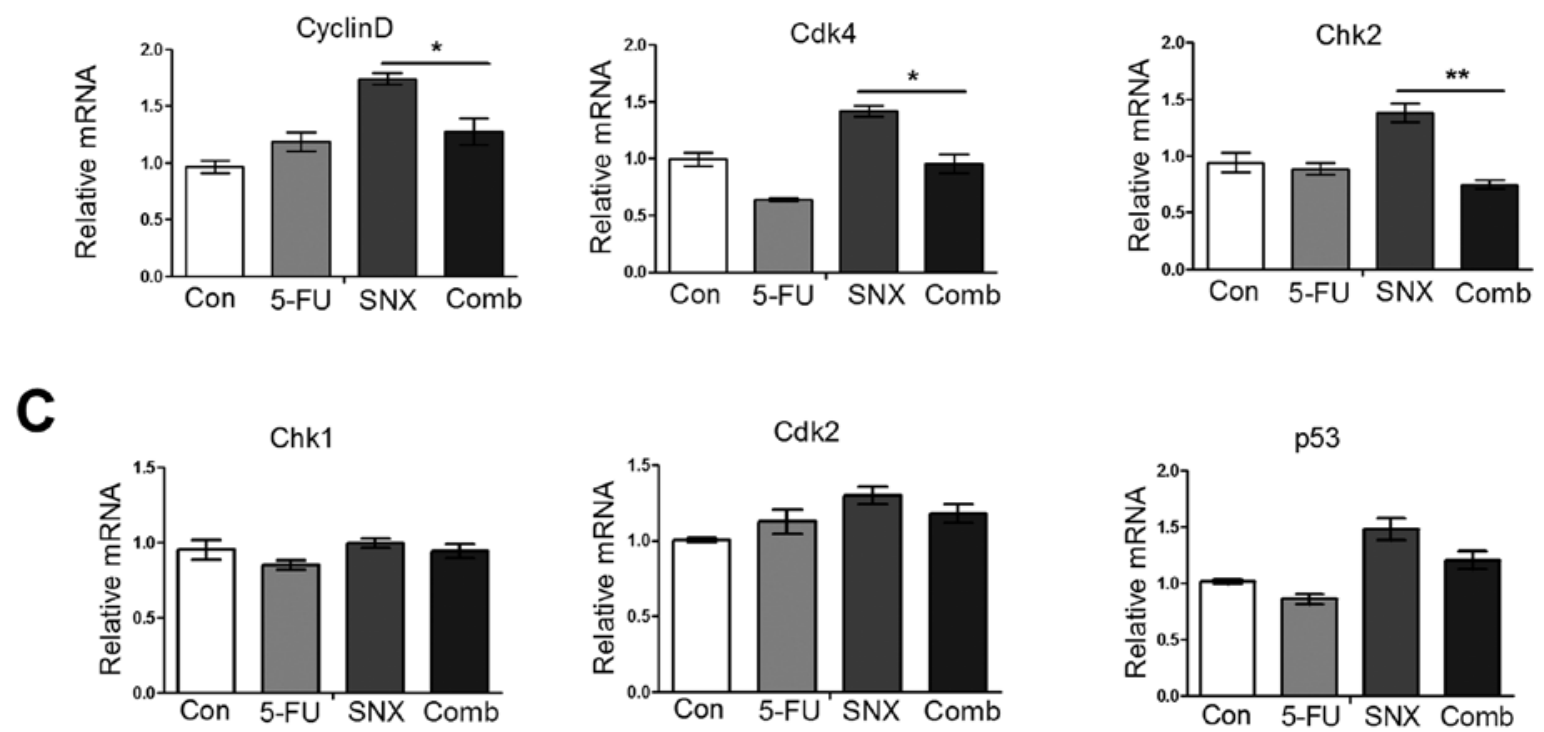

Figure 2. Cell cycle arrest of 5-FU and SNX-2112 in Eca-109 cells. (A) Representative image of flow cytometry analysis of cell cycle distribution. Statistical histograms indicate the percentage of cells in each phase. (B) Relative mRNA level of cyclin D1, CDK4 and Chk2. (C) Relative mRNA level of Chk1, CDK2 and $\mathrm{p} 53$. Values are the mean \pm SD of three independent experiments. ${ }^{*} \mathrm{P}<0.05,{ }^{* *} \mathrm{P}<0.01$ compared with the SNX-2112 group. Con, untreated control; SNX, SNX-2112; Comb, combination.

while caspase- 8 (a downstream indicator of the death receptor apoptotic pathway) was inhibited slightly (Fig. 4A). To determine whether the mitochondria mediated the 5-FU antagonism of SNX-2112-induced apoptosis, we evaluated MMP by JC-1 staining. Following SNX-2112 treatment, the red/green fluorescence ratio was significantly decreased to 0.97 , which 5-FU recovered to 1.45 (Fig. 4B). Flow cytometry showed 39.1\% JC-1 fluorescence following SNX-2112 treatment; that of the combination group was $21.8 \%$ (Fig. 4C). These results show that 5-FU partly reversed the MMP decline caused by SNX-2112, indicating that 5-FU antagonism of SNX-2112-induced apoptosis might occur through mitochondrial repair.

5-FU inhibits SNX-2112-induced downregulation of Hsp90 client proteins. Previously, we found that SNX-2112 caused depletion of Hsp90 client proteins such as Akt, p-Akt, IKK, $\mathrm{ERK}_{1 / 2}$, and GSK-3 $\beta$ (18). To determine whether 5-FU antago- nized the anticancer effect of SNX-2112 on Hsp90 client proteins, we investigated the expression of these proteins following SNX-2112 and 5-FU treatment. The SNX-2112 and 5-FU combination partly inhibited Akt, p-Akt, ERK $\mathrm{ER}_{1 / 2}$, and GSK-3 $\beta$ depletion and recovered the SNX-2112-induced IKK $\alpha$ downregulation completely (Fig. 5A). These results suggest that 5-FU attenuates SNX-2112-induced downregulation of Hsp90 client proteins in Eca-109 cells.

5-FU and SNX-2112 do not bind competitively to the Hsp90 $N$-terminal ATP pocket. To study whether 5-FU binding to the N-terminal ATP pocket of Hsp90 competed with that of SNX-2112, the fit was examine by docking studies. A hydrogen bond residue (Phe-138) and a side chain donor molecule (Lys58) were in contact with SNX-2112; 5-FU did not interact with Hsp90, and a major portion of Hsp90 was exposed to the solvent. The scoring value of SNX-2112 and 5-FU was -30.94 and $-12.28 \mathrm{kcal} / \mathrm{mol}$, respectively, indicating that $5-\mathrm{FU}$ could 
A

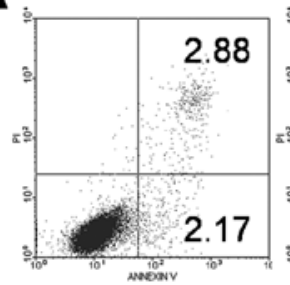

Control

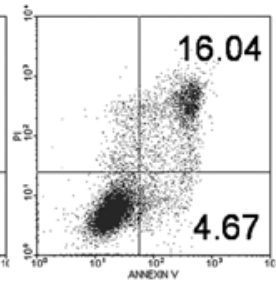

$5-\mathrm{FU}$

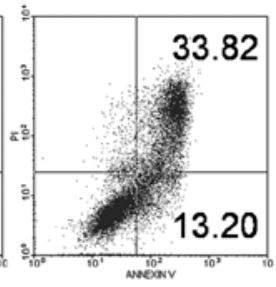

SNX-2112

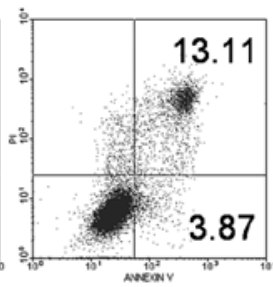

Combination

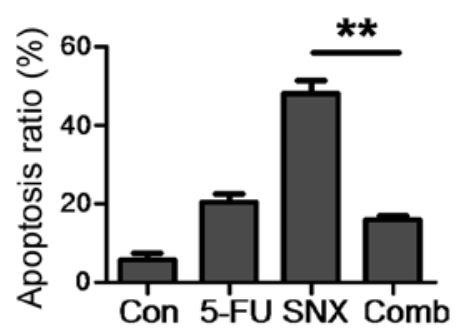

B
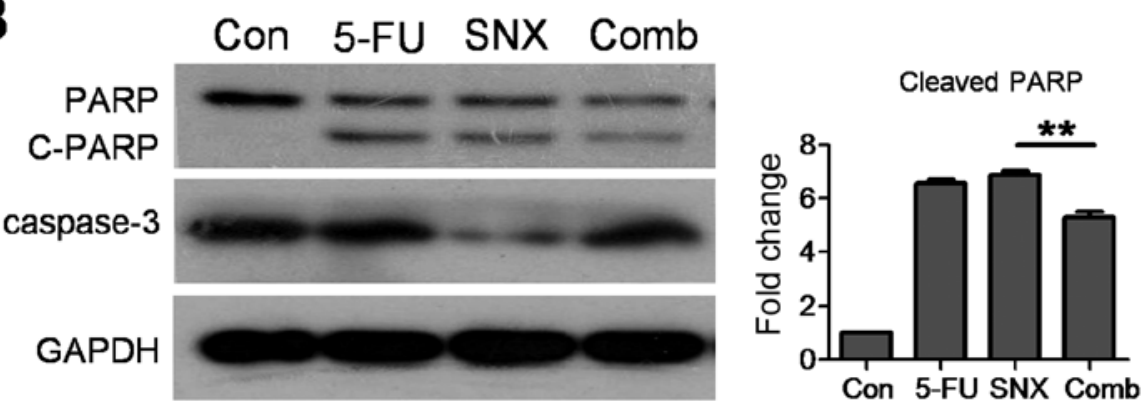

Caspase-3

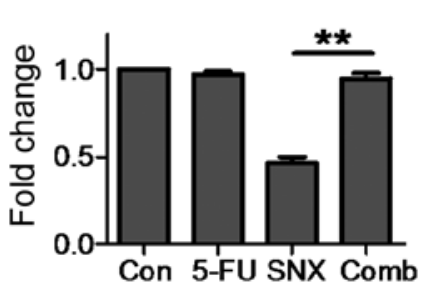

Figure 3. Caspase-dependent apoptosis after 5-FU and SNX-2112 treatment in Eca-109 cells. (A) Representative image of assessment of apoptosis by Annexin V/PI staining. Statistical histograms indicate the percentage of apoptotic cells. (B) Western blot assessment of apoptosis-associated proteins PARP and caspase-3. GAPDH was used as the protein loading control. Values are the mean $\pm \mathrm{SD}$ of three independent experiments. ${ }^{*} \mathrm{P}<0.05,{ }^{* * *} \mathrm{P}<0.01$ compared with the SNX-2112 group. Con, untreated control; SNX, SNX-2112; Comb, combination; C-PARP, cleaved PARP.

A
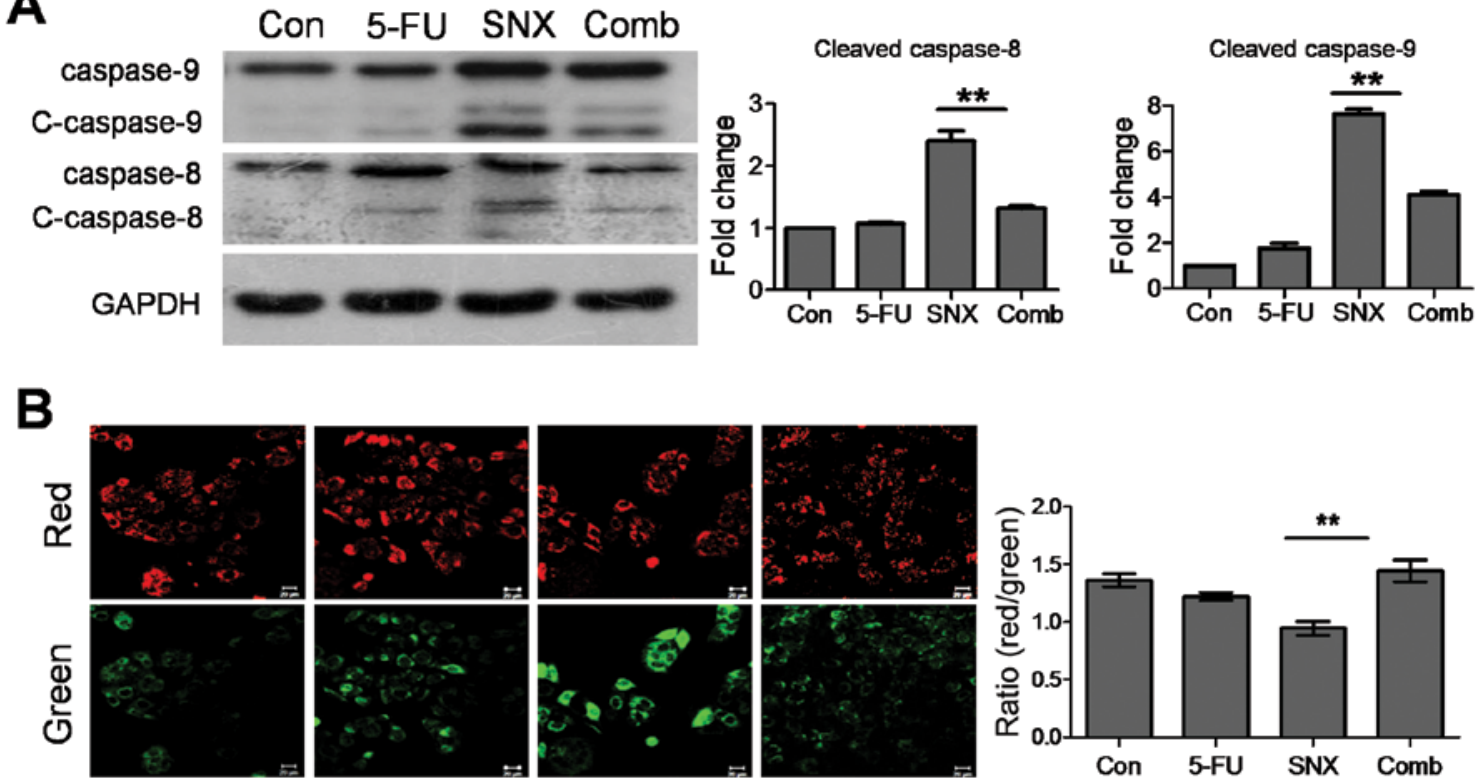

Control
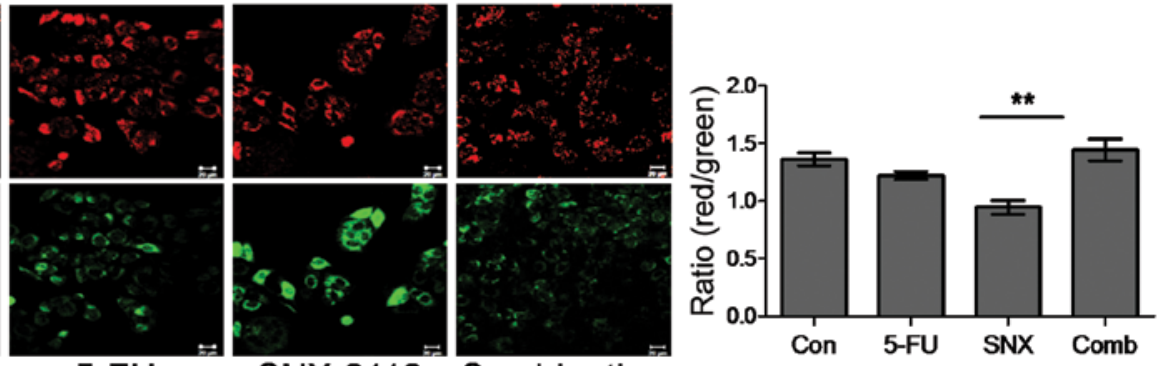

C
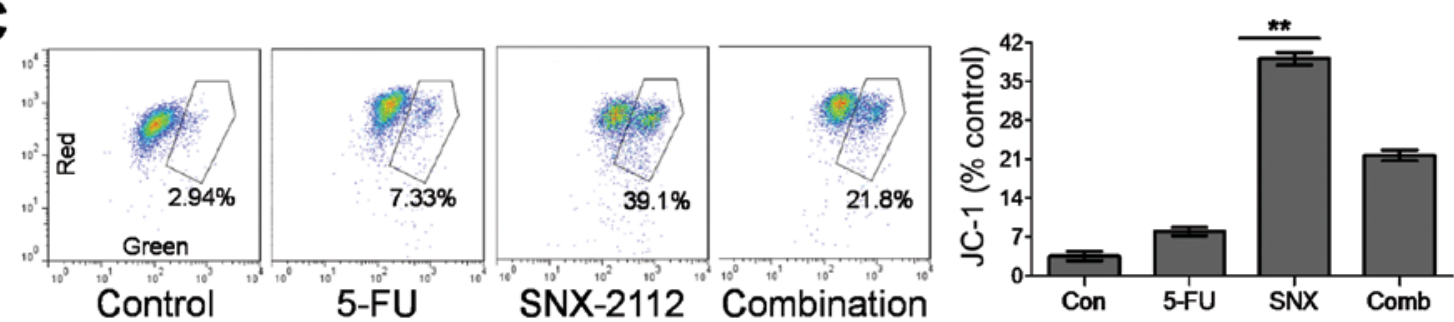

Figure 4. Effect of 5-FU and SNX-2112 on MMP in Eca-109 cells. (A) Western blot assessment of caspase-9 and -8. GAPDH was used as the protein loading control. (B) JC-1 fluorescence images of cells treated with SNX-2112, 5-FU, or SNX-2112 and 5-FU. Red fluorescence indicates high membrane potential; green fluorescence represents low membrane potential. (C) Representative flow cytometric analyses of MMP in cells treated with SNX-2112, 5-FU, or SNX-2112 and 5-FU; increased JC-1 expression indicates reduced MMP. Values are the mean $\pm \mathrm{SD}$ of three independent experiments. " $\mathrm{P}<0.05$, ${ }^{* * *} \mathrm{P}<0.01$ compared with the SNX-2112 group. Con, untreated control; SNX, SNX-2112; Comb, combination; C-caspase-8, cleaved caspase-8; C-caspase-9, cleaved caspase-9. 


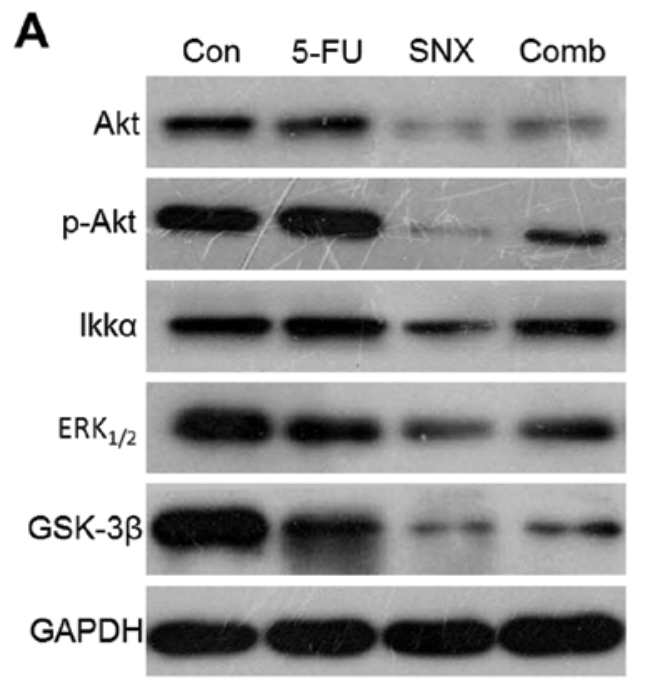

B
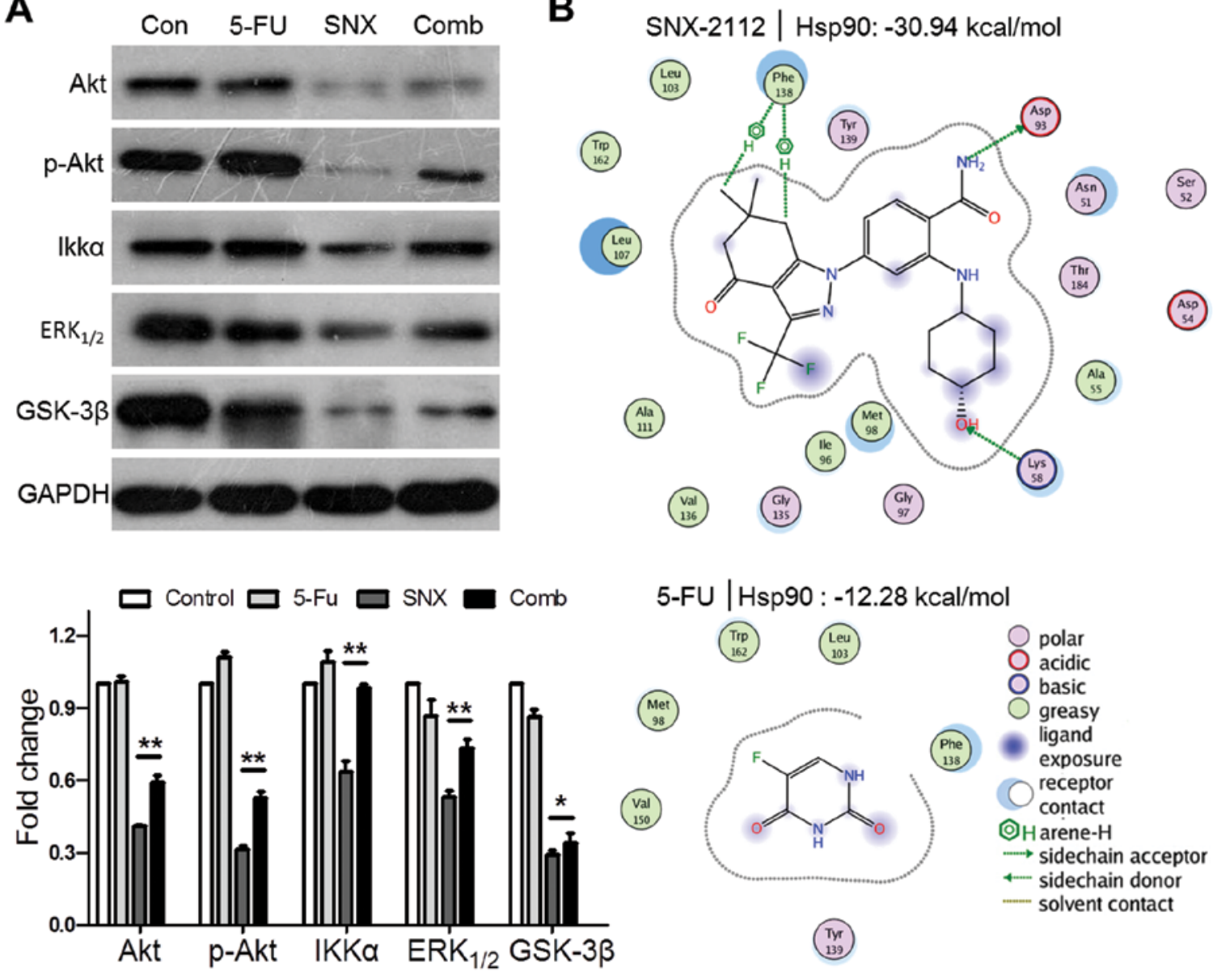

5-FU | Hsp90 : $-12.28 \mathrm{kcal} / \mathrm{mol}$

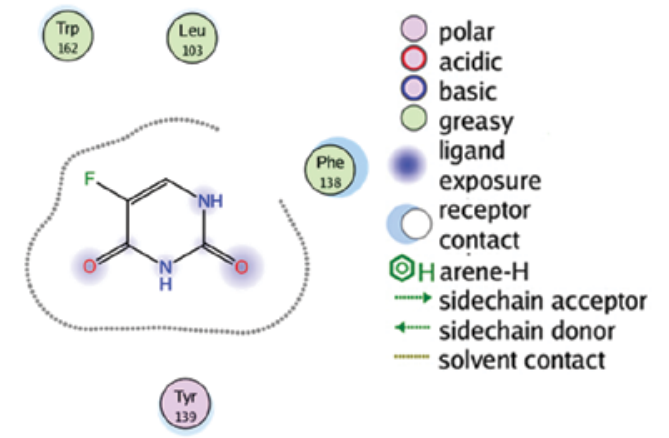

C

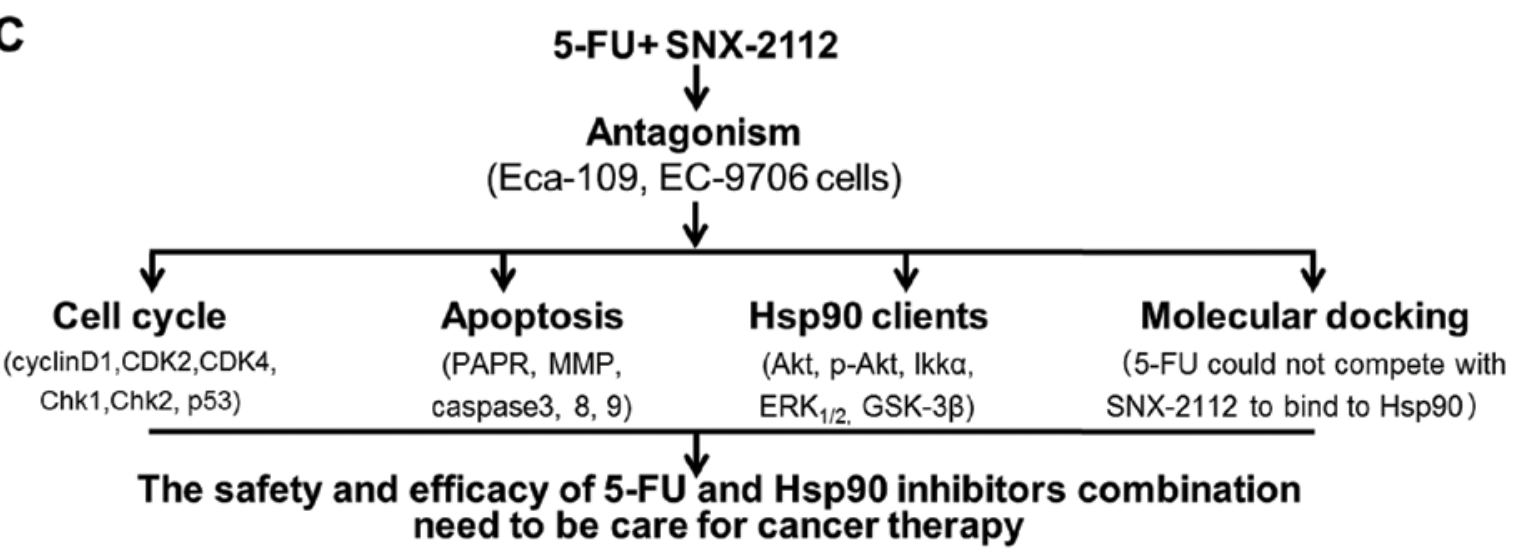

Figure 5. The safety and efficacy of the combination of 5-FU and Hsp90 inhibitors need special attention in cancer therapy. (A) Effects of SNX-2112, 5-FU,

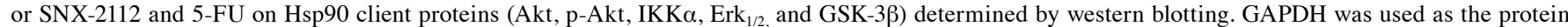
loading control. (B) 5-FU and SNX-2112 docking at the N-terminal domain of Hsp90. The lowest-energy conformations are shown; lower scores indicate more favorable binding. (C) Graphical abstract. Values are the mean \pm SD of three independent experiments. ${ }^{*} \mathrm{P}<0.05,{ }^{* *} \mathrm{P}<0.01$ compared with the SNX-2112 group. Con, untreated control; SNX, SNX-2112; Comb, combination.

not compete with SNX-2112 to bind to the N-terminal ATP pocket of Hsp90 (Fig. 5B).

\section{Discussion}

This study marks the first demonstration that the combination of SNX-2112 with 5-FU exhibits antagonistic effects in esophageal cancer cells. The antagonist effects were related to: i) growth inhibition; ii) G2/M arrest; iii) caspase-dependent and mitochondrial-mediated apoptosis; iv) Hsp90 client protein expression. In most cases, however, the combination of Hsp90 inhibitors with chemotherapy drugs (such as SNX-2112 with paclitaxel and cisplatin in head and neck SCC, ganetespib with the taxanes paclitaxel and docetaxel in non-small cell lung cancer, 17-AAG with cisplatin in glioma) is synergistic $(17,22,23)$. From this point of view, the combination effect of SNX-2112 plus 5-FU is quite different from that of other combination therapies based on Hsp90 inhibi- 
tors and chemotherapy drugs, and the antagonistic effect in this exception catalyzed our efforts to uncover the underlying mechanism.

SNX-2112 arrests the cell cycle at the G2/M phase in various cancers $(16,18)$. It has been established that cyclins, cell cycle kinases [such as checkpoint proteins, cyclin-dependent kinases (Cdks), and non-Cdk kinases], and other cell cycle-related protein are responsible for cell cycle control. For instance, Cdk4 is inactive in their monomeric form and activated by cyclin D1, and the formation of Cdk4/cyclin D1 complexes plays an important role on the change of cell cycle (24). Stepanova et al reported that the inactivation of Hsp90 increased the complexes of Cdk4/cyclin D1, but has no effect on Cdk2/cyclin E1 (25). These findings are consistent with our study that the mRNA level of cyclin D1 and Cdk4 were decreased in combination group compared to SNX-2112 treatment alone, with no significant change of Cdk2. In addition, we found that 5-FU completely reversed SNX-2112induced G2/M arrest and partly increased the G0/G1 arrest of SNX-2112. However, this contrasts with the 5-FU enhancement (but not decrease) of $\mathrm{G} 2 / \mathrm{M}$ cell percentage that may antagonize the effect of celecoxib in breast cancer (26). It remains unclear why 5-FU has bidirectional effects in cell cycle arrest when combined with different anticancer drugs.

We demonstrated that 5-FU suppressed caspase-dependent and mitochondria-mediated apoptosis induced by SNX-2112. Consistent with our previous studies, SNX-2112 activated caspase-3, -8 and -9 and decreased MMP (14-16,18), which 5 -FU neutralized in this study. Mitochondria are involved in many cellular processes, such as metabolism, signaling, cell growth, and apoptosis (21). Caspase-9 activation is significantly associated with mitochondrial dysfunction, while caspase- 8 activation is related to the death receptor pathways (27). In our protein assay, caspase- 9 appeared more sensitive than caspase- 8 to 5-FU treatment, suggesting that 5-FU antagonism of SNX-2112 is mainly regulated by mitochondrial-dependent pathways.

The exact manner in which 5-FU decreases the anticancer effect of the Hsp90 inhibitor SNX-2112 remains to be determined. However, based on the literature and our findings, we believe that there are at least two probabilities: i) 5-FU might bind competitively to the N-terminal ATP pocket or another site of Hsp90, altering Hsp90 conformation and function. However, it should be noted that our molecular docking study only ruled out the possibility of 5-FU indirect binding to the N-terminal ATP pocket of Hsp90; ii) 5-FU recovers the downstream signaling pathways of Hsp90 downregulated by SNX-2112. Our report proves that SNX-2112 suppresses the phosphatidylinositol 3-kinase (PI3K)/Akt and nuclear factor $\kappa \mathrm{B}(\mathrm{NF}-\mathrm{\kappa} B)$ pathways $(16,28)$. It has been reported that 5 -FU alone upregulates p-Akt and IKK expression in cancer cells $(29,30)$. Therefore, we speculate that the recovery of the PI3K/Akt and NF- $\mathrm{KB}$ pathways might be a possible mechanism of 5-FU antagonism of the anticancer effect of SNX-2112.

In conclusion, the combination of SNX-2112 with 5-FU exhibits antagonistic effects by reversing G2/M arrest, suppressing caspase-dependent apoptosis, preventing the initial MMP decrease, and decreasing the downregulation of
Hsp90 client proteins (Fig. 5C). Although further demonstration of a more precise mechanism of 5-FU antagonism of SNX-2112 is required in other cancer types, we suggest that the SNX-2112 plus 5-FU combination should be used with special care in clinical application.

\section{Acknowledgements}

This study was supported by grants from the National Natural Science Foundation of China (grant 81201727), the China Postdoctoral Science Foundation (grants 2012M511882 and 2013T60827), the open project of State Key Laboratory of Molecular Oncology (SKL-KF-2013-14), Guangdong Province and Ministry of Education Ministry of Science and Technology Products Research Combined Platform Project (grant 2010B091000013), and the Natural Science Foundation of Guangdong Province (grant S2012040006873).

\section{References}

1. Jemal A, Bray F, Center MM, Ferlay J, Ward E and Forman D: Global cancer statistics. CA Cancer J Clin 61: 69-90, 2011.

2. He YT, Hou J, Chen ZF, et al: Trends in incidence of esophageal and gastric cardia cancer in high-risk areas in China. Eur J Cancer Prev 17: 71-76, 2008.

3. Tew WP, Kelsen DP and Ilson DH: Targeted therapies for esophageal cancer. Oncologist 10: 590-601, 2005.

4. Khushalani NI, Leichman CG, Proulx G, et al: Oxaliplatin in combination with protracted-infusion fluorouracil and radiation: report of a clinical trial for patients with esophageal cancer. J Clin Oncol 20: 2844-2850, 2002.

5. Burmeister BH, Walpole ET, D'Arcy N, et al: A phase II trial of chemoradiation therapy with weekly oxaliplatin and protracted infusion of 5-fluorouracil for esophageal cancer. Invest New Drugs 27: 275-279, 2009.

6. Juergens RA and Forastiere A: Combined modality therapy of esophageal cancer. J Natl Compr Cancer Netw 6: 851-861, 2008.

7. Neckers L: Hsp90 inhibitors as novel cancer chemotherapeutic agents. Trends Mol Med 8 (Suppl 4): S55-S61, 2002.

8. Faried A, Sohda M, Nakajima M, Miyazaki T, Kato $\mathrm{H}$ and Kuwano H: Expression of heat-shock protein Hsp60 correlated with the apoptotic index and patient prognosis in human oesophageal squamous cell carcinoma. Eur J Cancer 40: 2804-2811, 2004.

9. Liu KS, Zhang Y, Ding WC, et al: The selective Hsp90 inhibitor BJ-B11 exhibits potent antitumor activity via induction of cell cycle arrest, apoptosis and autophagy in Eca-109 human esophageal squamous carcinoma cells. Int J Oncol 41: 2276-2284, 2012.

10. Liu XL, Xiao B, Yu ZC, et al: Down-regulation of Hsp90 could change cell cycle distribution and increase drug sensitivity of tumor cells. World J Gastroenterol 5: 199-208, 1999.

11. Wu X, Wanders A, Wardega P, et al: Hsp90 is expressed and represents a therapeutic target in human oesophageal cancer using the inhibitor 17-allylamino-17-demethoxygeldanamycin. Br J Cancer 100: 334-343, 2009.

12. Bao XH, Takaoka M, Hao H-F, et al: Antiproliferative effect of the HSP90 inhibitor NVP-AUY922 is determined by the expression of PTEN in esophageal cancer. Oncol Rep 29: 45-50, 2013.

13. Rajan A, Kelly RJ, Trepel JB, et al: A phase I study of PF-04929113 (SNX-5422), an orally bioavailable heat shock protein 90 inhibitor, in patients with refractory solid tumor malignancies and lymphomas. Clin Cancer Res 17: 6831-6839, 2011.

14. Wang X, Wang S, Liu Y, et al: The Hsp90 inhibitor SNX-2112 induces apoptosis of human hepatocellular carcinoma cells: the role of ER stress. Biochem Biophys Res Commun 446: 160-166, 2014.

15. Wang R, Shao F, Liu Z, et al: The Hsp90 inhibitor SNX-2112, induces apoptosis in multidrug resistant K562/ADR cells through suppression of Akt/NF-kappaB and disruption of mitochondriadependent pathways. Chem Biol Interact 205: 1-10, 2013.

16. Wang SX, Ju HQ, Liu KS, et al: SNX-2112, a novel Hsp90 inhibitor, induces G2/M cell cycle arrest and apoptosis in MCF-7 cells. Biosci Biotechnol Biochem 75: 1540-1545, 2011. 
17. Friedman JA, Wise $\mathrm{SC}, \mathrm{Hu} \mathrm{M}$, et al: HSP90 inhibitor SNX5422/2112 targets the dysregulated signal and transcription factor network and malignant phenotype of head and neck squamous cell carcinoma. Transl Oncol 6: 429-441, 2013.

18. Liu KS, Liu H, Qi JH, et al: SNX-2112, an Hsp90 inhibitor, induces apoptosis and autophagy via degradation of Hsp90 client proteins in human melanoma A-375 cells. Cancer Lett 318 180-188, 2012.

19. Chou T-C and Talalay P: Quantitative analysis of dose-effect relationships: the combined effects of multiple drugs or enzyme inhibitors. Adv Enzyme Regul 22: 27-55, 1984.

20. Chou T-C: Drug combination studies and their synergy quantification using the Chou-Talalay method. Cancer Res 70 : 440-446, 2010.

21. Kroemer G, Galluzzi L and Brenner C: Mitochondrial membrane permeabilization in cell death. Physiol Rev 87: 99-163, 2007.

22. Ohba S, Hirose Y, Yoshida K, Yazaki T and Kawase T: Inhibition of $90-\mathrm{kD}$ heat shock protein potentiates the cytotoxicity of chemotherapeutic agents in human glioma cells. J Neurosurg 112: 33-42, 2010.

23. Proia DA, Sang J, He S, et al: Synergistic activity of the Hsp90 inhibitor ganetespib with taxanes in non-small cell lung cancer models. Invest New Drugs 30: 2201-2209, 2012.

24. Casimiro MC, Crosariol M, Loro E, Li Z and Pestell RG: Cyclins and cell cycle control in cancer and disease. Genes Cancer 3 : 649-657, 2012.
25. Stepanova L, Leng X, Parker SB and Harper JW: Mammalian p50Cdc37 is a protein kinase-targeting subunit of Hsp90 that binds and stabilizes Cdk4. Genes Dev 10: 1491-1502, 1996.

26. El-Awady RA, Saleh EM, Ezz M and Elsayed AM: Interaction of celecoxib with different anti-cancer drugs is antagonistic in breast but not in other cancer cells. Toxicol Appl Pharmacol 255: 271-286, 2011.

27. Crow MT, Mani K, Nam Y-J and Kitsis RN: The mitochondrial death pathway and cardiac myocyte apoptosis. Circ Res 95: 957-970, 2004.

28. Okawa Y, Hideshima T, Steed P, et al: SNX-2112, a selective Hsp90 inhibitor, potently inhibits tumor cell growth, angiogenesis, and osteoclastogenesis in multiple myeloma and other hematologic tumors by abrogating signaling via Akt and ERK. Blood 113: 846-855, 2009.

29. Fukuyama R, Ng KP, Cicek M, et al: Role of IKK and oscillatory NFkappaB kinetics in MMP-9 gene expression and chemoresistance to 5-fluorouracil in RKO colorectal cancer cells. Mol Carcinog 46: 402-413, 2007.

30. Azuma M, Yamashita T, Aota K, Tamatani $T$ and Sato M: 5-Fluorouracil suppression of NF-KappaB is mediated by the inhibition of IKappab kinase activity in human salivary gland cancer cells. Biochem Biophys Res Commun 282: 292-296, 2001. 